\title{
Detection of Soilborne Disease Utilizing Sensor Technologies: Lessons Learned from Studies on Stem Rot of Peanut
}

\author{
Xing Wei, ${ }^{1,2}$ Marcela Aguilera, ${ }^{2,3}$ Rachael Walcheck, ${ }^{1,4,5}$ Dorothea Tholl, ${ }^{5}$ Song Li, ${ }^{2}$ David B. Langston, Jr., ${ }^{1,2}$ \\ and Hillary L. Mehl ${ }^{1,2,+}$ \\ ${ }^{1}$ Virginia Tech Tidewater Agricultural Research and Extension Center, Suffolk, VA 23437 \\ 2 School of Plant and Environmental Sciences, Virginia Tech, Blacksburg, VA 24061 \\ ${ }^{3}$ Genetics, Bioinformatics, and Computational Biology Program, Virginia Tech, Blacksburg, VA 24061 \\ ${ }^{4}$ Microbiology Undergraduate Program, lowa State University, Ames, IA 50011 \\ ${ }^{5}$ Department of Biological Sciences, Virginia Tech, Blacksburg, VA 24061
}

Accepted for publication April 12, 2021.

\section{Abstract}

Soilborne plant diseases are a major constraint to crop production worldwide. Effective and economical management of these diseases is dependent on the ability to accurately detect and diagnose their signs and/or symptoms prior to widespread development in a crop. Sensor-based technologies are promising tools for automated crop disease detection, but research is still needed to optimize and validate methods for the detection of specific plant diseases. The overarching goal of our research is to use the peanut-stem rot plant disease system to identify and evaluate sensor-based technologies that can be utilized for the detection of soilborne plant diseases.
Here we summarize the current state of sensor-based technologies for plant disease detection and provide examples from our own research that illustrate the advantages and limitations of different sensor-based methods for detecting soilborne diseases. In addition, the potential to adapt different sensor-based technologies to practical use in the field is discussed.

Keywords: optical sensors, spectral reflectance, thermal imaging, volatile organic compounds, machine learning, random forest, hyperspectral, Athelia rolfsii, Arachis hypogaea
Soilborne plant pathogens, which cause seedling, vascular, and root rot diseases, are detrimental to global food production (Ka$\tan$ 2017; Oerke 2006). These diverse pathogens, which include fungi, oomycetes, bacteria, viruses, and nematodes, share a common feature of spending at least part of their life cycle in the soil (Bruehl 1987; Katan 2017). Management of soilborne pathogens is challenging because the inoculum can survive in the soil without the crop host for long periods of time. In addition, these pathogens typically have a broad host range (Divya Rani and Sudini 2013), and it is laborious and time-consuming to

\section{${ }^{\dagger}$ Corresponding author: H. L. Mehl; hillary.mehl@usda.gov}

Current address for H. L. Mehl: United States Department of Agriculture, Agricultural Research Service, Arid-Land Agricultural Research Center, Tucson, AZ 85701 .

Mention of trade names or commercial products in this article is solely for the purpose of providing specific information and does not imply recommendation or endorsement by the U.S. Department of Agriculture. USDA is an equal opportunity provider and employer.

Funding: Studies on spectral and thermal sensors were funded in part by Virginia Peanut Board and Virginia Agricultural Council. The VOC detection study was a research project in the Research and Extension Experimental Learning (REEL) program at Virginia Tech funded by the Agriculture and Food Research Initiative program of the National Institute of Food and Agriculture, USDA (grant no. 201767032-26017)

The author(s) declare no conflict of interest.

(c) 2021 The American Phytopathological Society manually detect and identify specific diseases in the field. Inexpensive and reliable disease detection, identification, and quantification are needed to optimize disease control strategies, help growers make management decision, and maximize net profits (Bock et al. 2010; Nutter 1990; Nutter et al. 1991, 1993). Sensor technologies are a promising tool for automated plant disease detection, identification, and quantification, and this is a rapidly expanding area of research (Mahlein 2016; Nutter 1990; Oerke 2020).

Sensors that can potentially be used for plant disease identification fall into two general categories: optical and nonoptical (Fig. 1) (Oerke et al. 2014). Various types of optical sensors have been used for plant disease detection. These include RGB, multi- and hyperspectral reflectance, thermal, fluorescence, and light detection and ranging (LiDAR) sensors (Mahlein 2016; Oerke 2020). Among these sensors, spectral reflectance and infrared thermography are of particular interest in plant disease detection and plant phenotyping because they can be utilized with ground-based, airborne, and satellite-based platforms (Mahlein 2016; Oerke et al. 2014). Among the nonoptical type sensors, those that can detect volatile organic compounds (VOCs) emitted by the plant and/or pathogen during disease development have been utilized the most for plant disease detection. These include but are not limited to VOC-trapping devices coupled with gas chromatography-mass spectrometry (GC-MS), electronic noses (e-nose), and other novel VOC sensors ( $\mathrm{Li}$ et al. 2019; Oerke 2020). Although there has recently been an exponential increase in research aimed at utilizing various sensor technologies for plant disease detection, additional studies and 
technological improvements aimed at identifying and detecting disease-specific signatures are needed before they can be used as a practical tool for in-field plant disease detection and site-specific management.

The sensitivity and specificity of sensor-based methods, which highly depend on spatial resolution, are critical to detect and identify plant diseases caused by microorganisms (Oerke et al. 2014). The spatial resolution, referring to the minimum size of a pixel in image-based optical sensors, relies on the distance between the sensor and the object (Mahlein 2016; Oerke et al. 2014). Proximal sensing platforms are suitable for disease detection at leaf and plant scale, whereas remote sensing with airborne platforms is useful for detecting soilborne disease patches in the field or in later stages of disease epidemics (Hillnhütter et al. 2011; Mahlein et al. 2012; Nutter et al. 2010; Oerke et al. 2014). In addition to spatial resolution, Hillnhütter and Mahlein (2008) suggested spectral resolution and temporal factors are also essential for detecting small areas in a field, a critical capacity during the early stage of disease development.

Currently, one of the major limitations to using sensors for plant disease detection is the lack of an efficient method to analyze and interpret the great amount of complex data generated with sensor measurements (Mahlein 2016; Moran et al. 1997). Machine learning algorithms are a very promising approach that can be used to collate and statistically evaluate large amounts of sensor data more efficiently and without explicit programing (Samuel 1959; Singh et al. 2016). Along with sensor technologies, machine learning approaches have been increasingly applied in precision crop management for yield prediction and detection of abiotic and biotic stresses such as pest insects, diseases, and weeds (Liakos et al. 2018; Mahlein et al. 2018). Machine learning algorithms are capable of generalizing trends or patterns from large amounts of data efficiently (Singh et al. 2016), which is essential for timely application of crop management practices based on sensor technologies.

\section{Exploring Sensor-Based Methods to Detect Stem Rot of Peanut}

Stem rot, caused by Athelia rolfsii (anamorph Sclerotium rolfsii), is one of the most economically important soilborne diseases of peanut (Arachis hypogaea L., also known as groundnut) worldwide. Yield loss caused by this disease is typically less than $25 \%$ but can be up to $80 \%$ under conditions favorable to $A$. rolfsii, which include warm to hot temperatures $\left(27\right.$ to $\left.30^{\circ} \mathrm{C}\right)$ and high relative humidity (>90\%) (Backman and Brenneman 1997; Punja 1985). The initial symptoms exhibited by peanut plants infected with $A$. rolfsii are water-soaked necrotic lesions on stems, roots, pegs, or pods, and the foliar symptoms include wilting of a single lateral branch, the main stem, or the entire plant (Fig. 2) (Backman and Brenneman 1997). Signs of the pathogen include white appressed mycelium and brown round sclerotia, which are usually found at or near the soil line on and around the affected plant parts (Fig. 2).

Foliar-applied fungicides are used for management of stem rot, but they are most effective when applied prior to or during the early stages of infection. As with other soilborne crop diseases, scouting via visual inspection is frequently used to detect signs and symptoms of the disease and make management decisions. However, with the expansion of data-based, site-specific crop management, there is a need and demand for automated sensor-based plant disease detection. Using the peanut-stem rot plant disease system, the overarching goals of our research are to (i) identify and evaluate multiple sensor-based technologies that can be utilized for detection of soilborne plant diseases and (ii) explore the benefits and limitations of each, including their

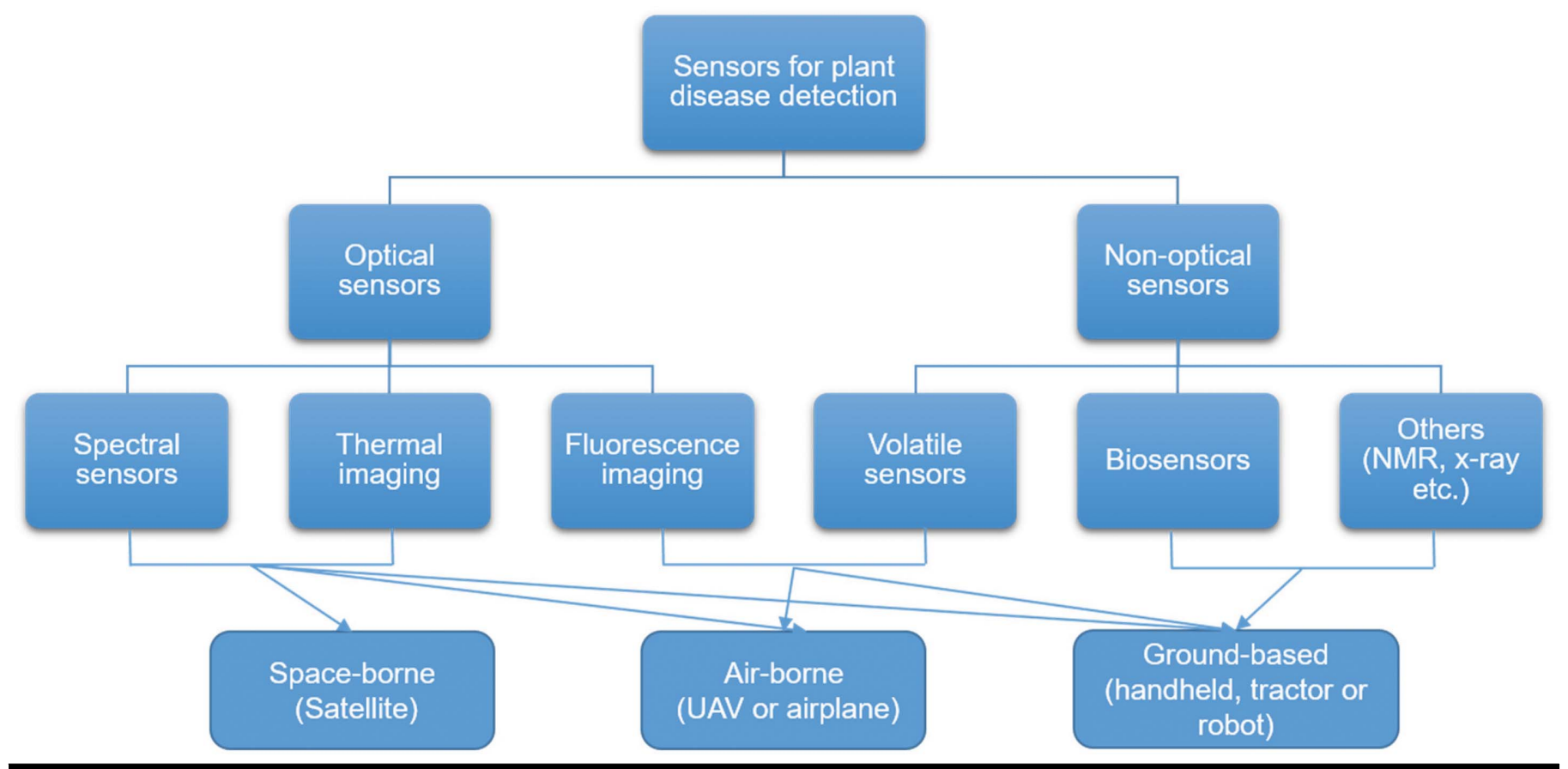

FIGURE 1

Commonly used sensors for plant disease detection and their application platform (adapted based on information from Fang and Ramasamy 2015, Mahlein 2016, and Oerke et al. 2014). Volatile sensors include portable gas chromatography-mass spectrometry systems and electronic noses (e-nose). Biosensors are sensors based on highly selective biorecognition elements such as enzyme, antibody, DNA-RNA, and bacteriophage (Fang and Ramasamy 2015). NMR = nuclear magnetic resonance, and UAV = unmanned aerial vehicle. 
relative sensitivity and specificity as well as potential to be adapted to field use.

\section{Spectral Signatures of Peanut Plants Infected with}

\section{A. rolfsii}

Greenhouse experiments were conducted at the Virginia Tech Tidewater Agricultural Research and Extension Center in Suffolk, VA, to identify responses of peanut to infection with $A$. rolfsii under controlled conditions (Wei et al. 2019, 2020, 2021). Approximately 70-day-old 'Sullivan' peanut plants were either inoculated with $A$. rolfsii or mock inoculated via a clothespin technique adapted from Shokes et al. (1996). Briefly, clothespins colonized with $A$. rolfsii were clamped on lateral stems of peanut plants (Fig. 3A). Noncolonized clothespins were clamped on mock-inoculated control stems. In each experiment, inoculated lateral stems were compared with mock-inoculated controls. Following inoculation, plants were placed inside a moisture chamber to facilitate pathogen infection and disease development. Starting 2 days after inoculation, plants were inspected daily for visual disease symptoms. The rating scale of disease severity was adapted from Shokes et al. (1996), where $1=$ healthy without any disease symptoms, $2=$ necrotic lesions on the inoculated stems only, $3=$ up to $50 \%$ of leaves on the inoculated stems symptomatic (discoloration, prevalent veins, and wilting), $4=$ more than $50 \%$ of leaves symptomatic, and $5=$ withering of whole inoculated stems. Inside the greenhouse, plants were placed outside the moisture chamber during daily measurement and were moved back to the moisture chamber after the measurement was completed.

Spectral reflectance of a designated leaflet on the second youngest leaf on the treated lateral stem was measured daily by a SpectroClip-R probe with a handheld Jaz spectrometer (Fig. $3 \mathrm{~B})$, and a machine learning approach was used to analyze the resulting data and identify stem rot-associated spectral signatures. A total of 252 spectra were selected from the greenhouse experiments and assigned into three classes based on disease severity ratings: healthy ( scale $=1$ ), mildly symptomatic (scale $>2$ but $<4$ ), or severely diseased (scale $\geq 4$ ) based on visual symptomology. Spectral data were analyzed using the R statistical platform with methods adapted from Heim et al. (2018). Extreme ends of spectra were deleted due to noisy signals, and outlying spectra were removed using depth measures (FebreroBande and Oviedo de la Fuente 2012). First-order derivatives of each spectrum were also calculated to minimize random noise, thus improving classification quality (Demetriades-Shah et al. 1990). The random forest algorithm was used for both the classification and feature selection of important wavelengths (Breiman 2001; Genuer et al. 2015; Kuhn et al. 2017). The spectral dataset was randomly split 80:20 into training and testing subsets. The training data were used to train a random forest classifier to learn

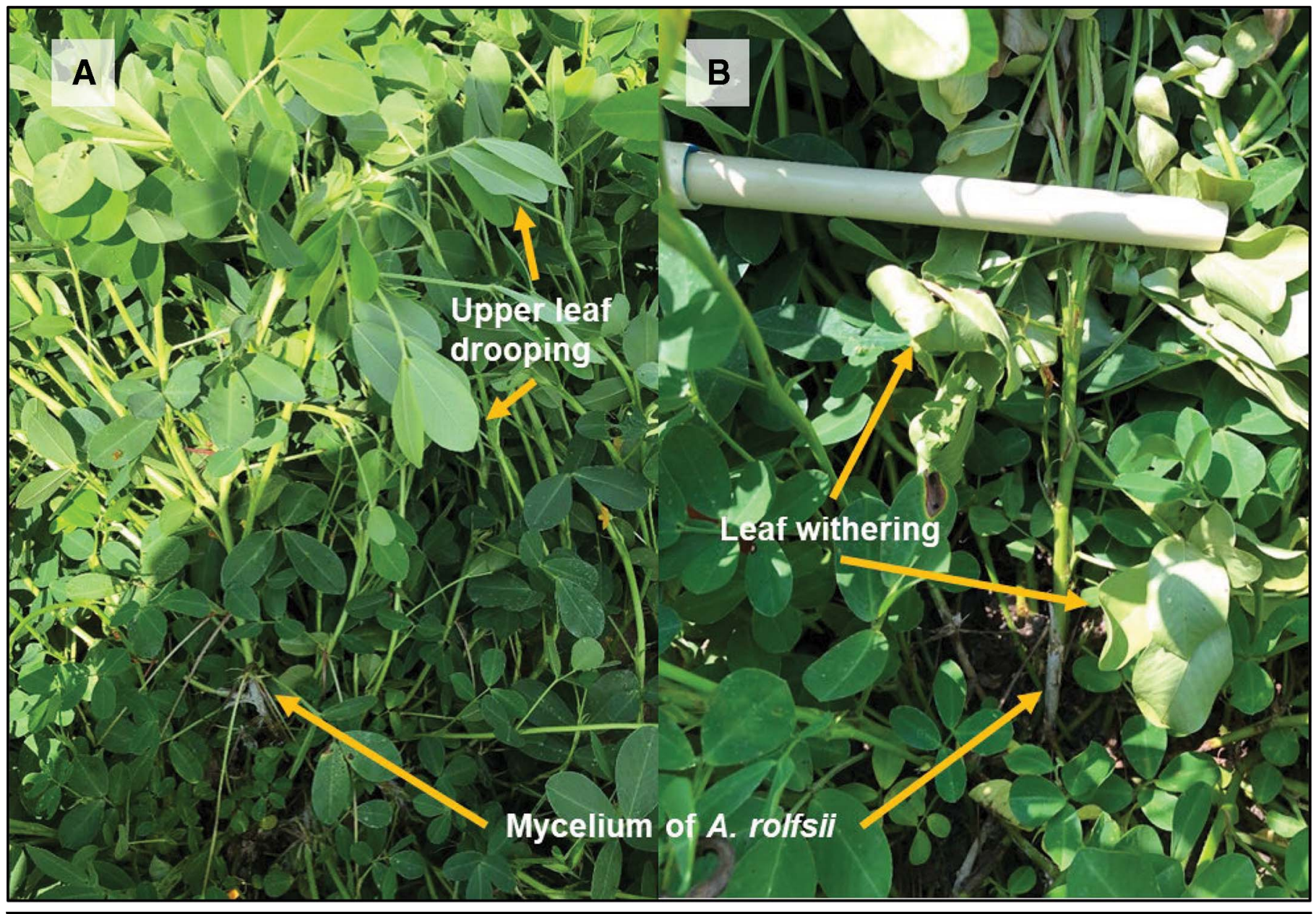

FIGURE 2

Signs and symptoms of peanut plants naturally infected with Athelia rolfsii in the field during $\mathbf{A}$, early and $\mathbf{B}$, late infection stage. 
patterns to distinguish between healthy and diseased plants, and the testing data were used to evaluate how correctly the trained model could classify "unknown" spectra as coming from healthy versus diseased plants.

Overall, leaves of peanut plants infected with $A$. rolfsii exhibited greater reflectance compared with noninfected healthy plants in both visible and near-infrared regions (Fig. 4), suggesting that infection of peanut stems with A. rolfsii results in alteration of pigment concentrations and cell structure of the peanut leaves. Using a machine learning algorithm, it was possible to distinguish healthy, noninfected peanut plants from symptomatic $A$. rolfsii-infected plants with an overall accuracy greater than $80 \%$ (Table 1). Most wavelengths selected by the random forest algorithm as indicative of plant health were from the visible region (Fig. 4), which agreed with the three visual observation classes of foliar symptoms: "healthy", "mild", and "severe". Additional studies are needed to determine if these wavelengths represent disease-specific signatures of $A$. rolfsii infection in peanuts.

\section{Thermal Response of Peanut Infected with A. rolfsii}

Thermal responses of peanut plants in the greenhouse experiments described above were measured using a forward-looking infrared camera at $\sim 183 \mathrm{~cm}$ above the ground inside the greenhouse. When taking thermal images, plants were placed on a wood board covered with a white needled cotton batting to provide a uniform thermal background (Fig. 3C). Soil in each plastic pot was also covered with the white cotton batting. Thermal images were taken daily following inoculation with $A$. rolfsii to measure changes in canopy temperature as the disease developed over time.

Leaflets on the $A$. rolfsii-inoculated stems had higher temperatures than leaflets on the mock-inoculated and untreated stems at 8 and 12 days after inoculation (Fig. 5B and C). The higher leaf temperature in A. rolfsii-infected plants might be due to the reduced water supply to the leaves and reduced evapotranspiration cooling due to fungal colonization of the vascular system of inoculated stems (Nutter 1990; Tu and Tan 1985). Detection of temperature differences between inoculated and noninoculated peanut stems coincided with the observation of drooping of the terminal leaves on the

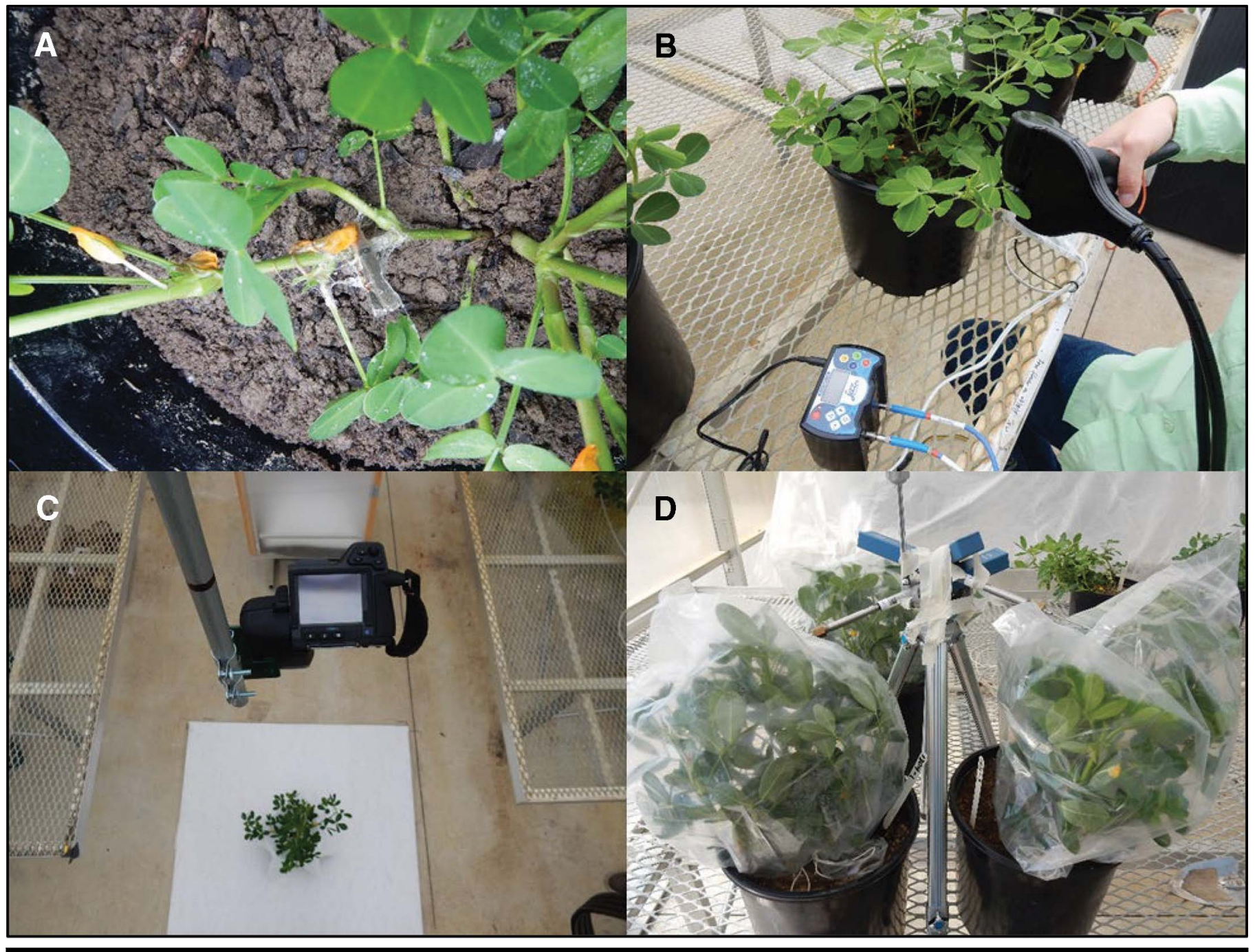

FIGURE 3

A, Peanut plants were inoculated via clamping a with Athelia rolfsii-colonized clothespin on one of the two major lateral stems in each plant. B, Spectral reflectance of one designated leaflet of the second youngest mature leaf on treated lateral stems was measured with a SpectroClip-R probe using a handheld Jaz spectrometer. C, Top-view thermal images of the whole plant were taken with a forward-looking infrared T420 camera at $\sim 183 \mathrm{~cm}$ above the ground inside the greenhouse. D, Volatile compounds were collected from the headspace of peanut plants enclosed in an oven bag using a pump with a charcoal trap. 
inoculated stems. However, temperature differences between inoculated and noninoculated stems were not consistent across all evaluation dates due to variability in environmental conditions during sensor measurements (Wei et al. 2021).

\section{Volatile Compounds Emitted by A. rolfsii-Infected Peanut Plants}

Peanut plants were inoculated in the greenhouse in order to identify VOCs released during early infection of peanut with $A$. rolfsii. Volatiles were collected from mock-inoculated control plants and plants that were inoculated with $A$. rolfsii 4 and 8 days prior to volatile collection. To inoculate/mock-inoculate peanut plants, 15-cm-diameter plugs of 3-day-old inoculated and noninoculated agar pieces were placed on the base of the main stem of each plant. Each plant was enclosed in an oven cooking bag and tied shut with a string under the inoculation site to concentrate the emitted volatiles. VOCs were collected for $24 \mathrm{~h}$ from the headspace of each plant using small pumps that pull volatiles on a charcoal trap (Fig. 3D). VOCs were then eluted from the traps with dichloromethane and analyzed by GC-MS.
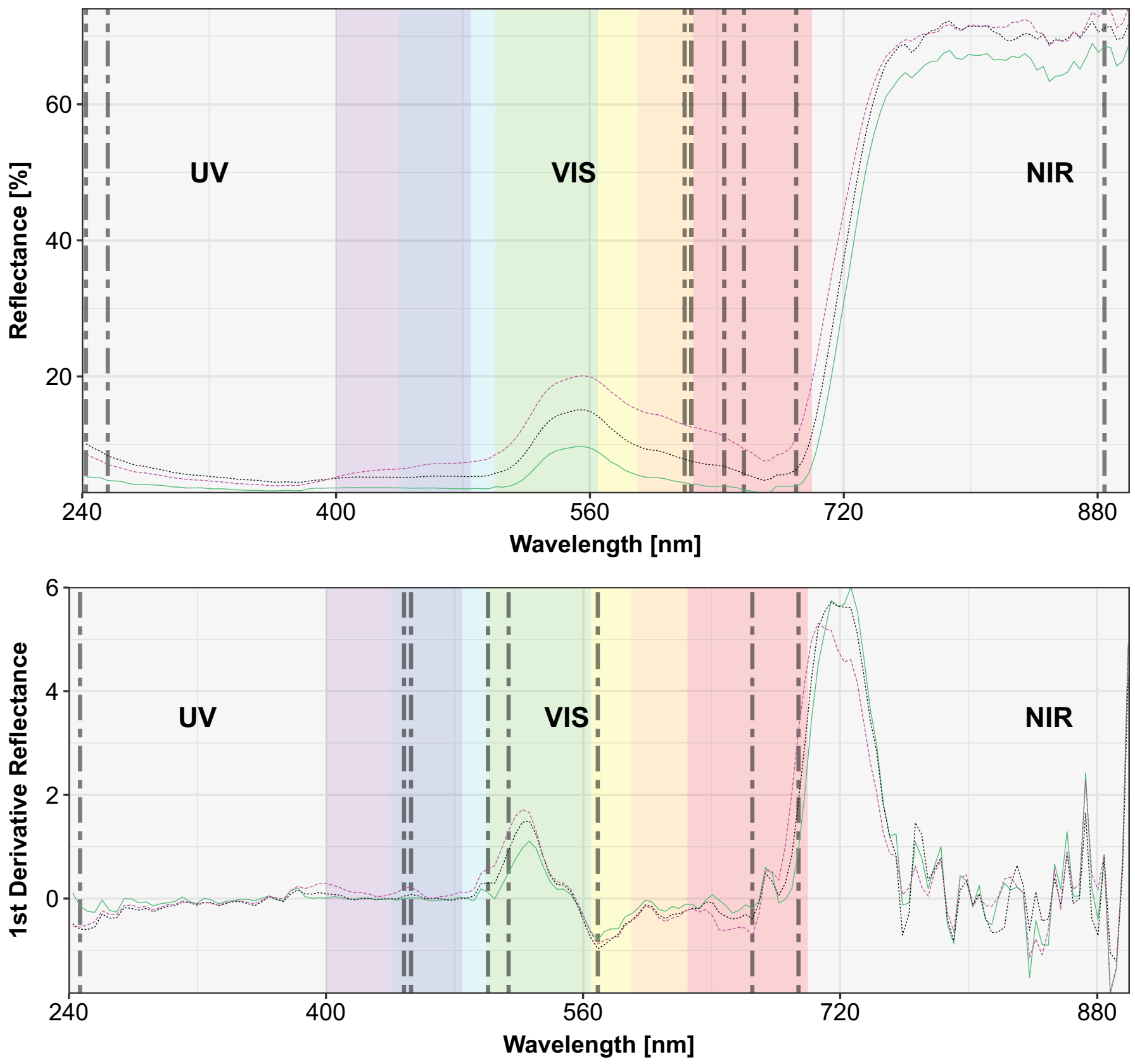

Type — Healthy ….. Mild …- Severe

FIGURE 4

Spectral signatures and selected wavelengths (as shown in drop lines) using the primary (top) and derivative-transformed (bottom) spectra of Athelia rolfsiiinoculated and mock-inoculated peanut plants. UV = ultraviolet; VIS = visible; and NIR = near-infrared. 
Emissions of compounds including hexenyl acetate, monoterpenes, the $\mathrm{C}_{16}$ homoterpene (E,E)-4,8,12-trimethyltrideca-1,3,7, 11-tetraene (TMTT) and especially the $\mathrm{C}_{11}$ homoterpene $(E)-4,8$ dimethyl-1,3,7-nonatriene (DMNT) were elevated in A. rolfsiiinfected plants 8 days after inoculation (Fig. 6). DMNT and TMTT are common VOCs that are released by angiosperms in response to herbivory and pathogen infection (Attaran et al. 2008; Tholl et al. 2011). DMNT has been previously reported as the predominant compound emitted from peanut plants infected with $A$. rolfsii. The homoterpene also occurs in volatile blends released from peanut damaged by the beet armyworm (Spodoptera exigua) and in response to a combination of both pathogen and herbivore attack (Cardoza et al. 2002). However, insect damage causes a substantial emission of several other compounds besides DMNT, which distinguishes this volatile blend from that induced only by fungal infection (Cardoza et al. 2002).

\section{Advantages and Limitations of Different Sensor-Based Methods for Detection of Soilborne Diseases}

Here, we define the sensitivity of a sensor-based method for plant disease detection as being indicative of how early the method can detect the pathogen infection and disease development. A highly sensitive method can detect initial pathogen infection prior to visible disease symptoms. Medium sensitivity detects the disease when symptoms just become visible. Low sensitivity methods can only detect the disease when severe symptoms have developed. We define the specificity of a sensorbased method for plant disease detection as how accurately the method can identify a specific pathogen infection and subsequent disease development. High-specificity methods can identify a specific disease with an accuracy greater than $80 \%$. Low specificity indicates the method can distinguish healthy plants and plants under stress but cannot determine whether plants are under abiotic or biotic stress. Methods with medium specificity fall in between these two.

In our studies, we conducted a preliminary evaluation of three sensor-based methods for the detection of stem rot of peanut. With all three methods, it was possible to detect responses of peanut plants to infection with $A$. rolfsii, but the sensitivity and specificity of the sensor measurements varied. Spectral reflectance differed between $A$. rolfsii-infected and noninfected peanut plants, and spectral signatures identified through machine learning algorithms were able to distinguish noninfected peanut plants from those with severe stem rot symptoms. However, the algorithm was less accurate for distinguishing noninfected plants from those with mild symptoms. Thus, the ability of spectral reflectance to accurately detect stem rot during the early stages of disease development is questionable. Foliar diseases (e.g., powdery mildew, rust, and leaf spots) typically have disease-specific symptoms, whereas symptoms of soilborne diseases might be confounded with abiotic stress such as drought and heat stresses. Nevertheless, hyperspectral sensors have demonstrated the potential to detect diseased plants before visible symptoms developed in several other plant disease systems (Arens et al. 2016; Gold et al. 2020b; Zarco-Tejada et al. 2018) and to distinguish different diseases in various crops (Gold et al. 2020a; Hillnhütter et al. 2011; Mahlein et al. 2013). Thus, overall spectral sensors were rated with a "medium" sensitivity and "high" specificity in the potential of identifying soilborne diseases (Table 2).

Thermal responses of peanut to the infection of $A$. rolfsii were detected when foliar symptoms became visible, but these thermal responses were not consistent across all the evaluation dates and were highly influenced by environmental conditions such as air temperature, humidity, and solar radiation (Wei et al. 2021).

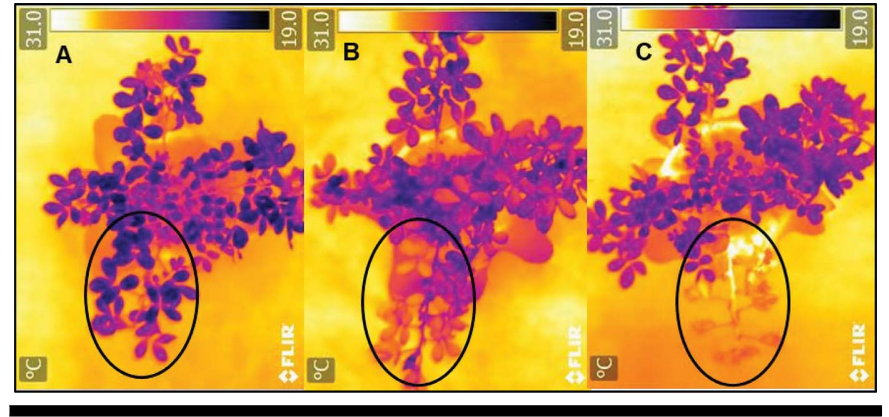

\section{FIGURE 5}

Thermal images of a peanut plant inoculated with Athelia rolfsii in a greenhouse experiment. The lateral stem inoculated with $A$. rolfsii is circled in each thermal image, whereas the opposite lateral stem was mock inoculated. $\mathbf{A}$, Four days after inoculation (DAl); B, 8 DAl; and C, 12 DAl. Darker color indicates cooler temperature, and lighter color indicates warmer temperature. Images were taken with a forward-looking infrared (FLIR) T420 camera with a top view at $\sim 183 \mathrm{~cm}$ above the ground inside the greenhouse.

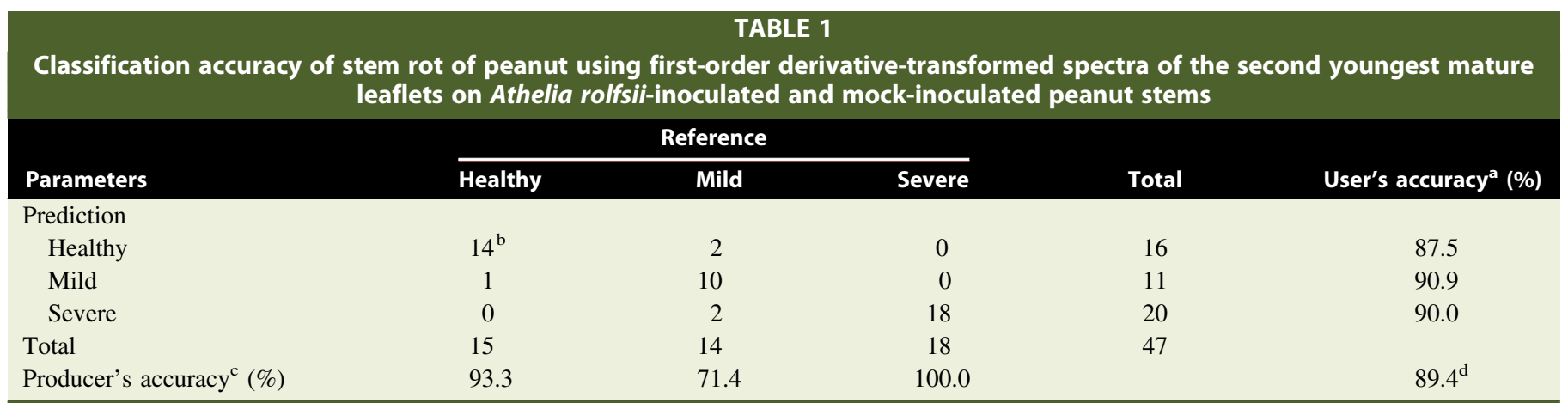

${ }^{a}$ User's accuracy was the number of correctly classified samples divided by the total number of prediction samples in each class. It represents the reliability of the classification accuracy for each specific class.

${ }^{\mathrm{b}}$ Numbers in the diagonals were the correctly classified samples, while off-diagonals were misclassified.

${ }^{\mathrm{c}}$ Producer's accuracy was the number of correctly classified samples divided by the total number of reference samples in each class. It represents the classification accuracy for each specific class.

${ }^{\mathrm{d}}$ Overall accuracy was the total number of correctly classified samples divided by the total number of samples. It represents the overall agreement between the reference and prediction. 
These results agree with previous studies, which suggest that thermal sensors are highly sensitive in detecting the leaf temperature changes due to changes in plant transpiration rate and water status caused by abiotic and biotic stresses (Chaerle and Van der Straeten 2000; Oerke and Steiner 2010; Oerke et al. 2014). However, the potential of using thermal sensors to identify a specific plant disease is limited because different stresses may alter the plant physiology similarly (Nutter et al. 2010), and thermal sensors are highly sensitive to environmental conditions (Oerke et al. 2014). Thus, thermal sensors were rated with a "medium to high" sensitivity and "low" specificity in the potential of identifying soilborne diseases (Table 2).

Results in previous studies and ours showed that VOCs could be detected from the pathogen itself (Fravel et al. 2002) and plants during the pathogenesis of pathogen infection (Cardoza et al. 2002). The VOCs collected in A. rolfsii-infected peanut plants were reported in peanut plants under other biotic stresses (Cardoza et al. 2002; Fravel et al. 2002) and in other plant systems (Boué et al. 2003; Zhang et al. 2008). Although VOC blends differ between pathogen and insect attack (Cardoza et al. 2002), overlap of VOC mixtures in response to multiple stressors makes it more difficult to immediately distinguish one from the other. Thus, the volatile-based detection method was rated with a "high" sensitivity and "low to medium" specificity in detecting soilborne plant diseases (Table 2).

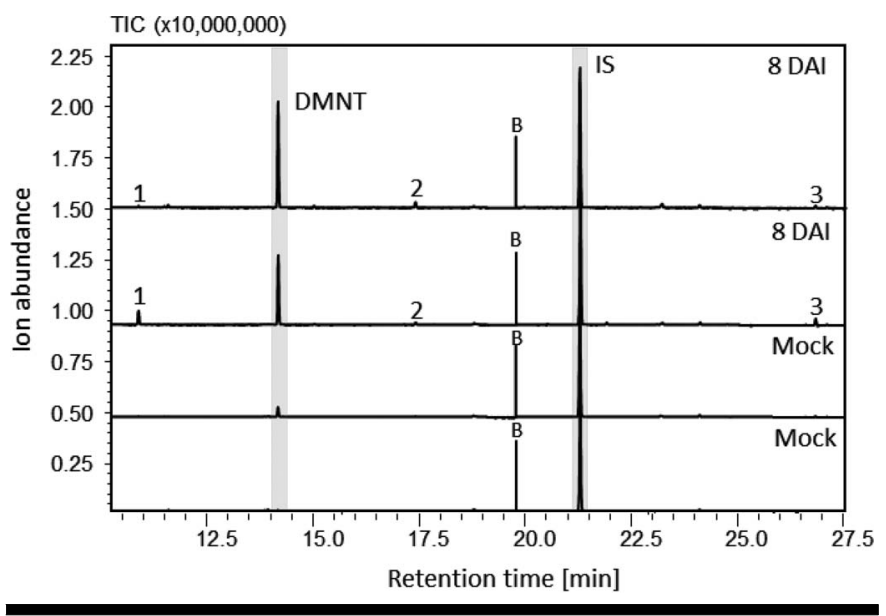

FIGURE 6

Volatile emission of peanut upon infection with Athelia rolfsii. GC-MS chromatograms of volatile organic compounds collected from shoots and leaves of $A$. rolfsii-infected peanut plants 8 days after inoculation (DAI) in comparison with mock-inoculated plants. DMNT $=(E)-4,8$-dimethyl-1,3,7nonatriene; 1 = hexenyl acetate; $2=$ putative monoterpene; $3=(E, E)-4,8$, 12-trimethyltrideca-1,3,7,11-tetraene; IS = internal standard; B = background; and TIC = total ion chromatogram. Identification of compounds is based on library suggestion.
Currently, there are various types of spectral and thermal sensors available on the market that can be handheld or mounted on ground-based tractors and robotic systems, airplanes, and satellites. The technical innovation and improvement of spectral and thermal sensors make them also suitable for mounting on unmanned aerial vehicles (UAVs) for large-scale mobile mapping applications (https://sphereoptics.de/en/). Therefore, the potential of field application of spectral and thermal sensors is high if the specific disease signatures (spatial and temporal) could be identified. Recent studies demonstrated the detection of several soilborne diseases with spectral sensors mounted on the UAV (Wang et al. 2020a, b) or satellite (Raza et al. 2020). Aerial infrared thermography has proved useful in characterizing and monitoring the spatial and temporal pattern of soilborne diseases for field application (Pinter et al. 1979; Powell et al. 1976; Schmitz et al. 2004). In addition, Oerke et al. (2014) suggested thermal sensors could be used in screening disease-resistance germplasms in the inoculated experiments in which identification of the specific disease is not necessary. The advantages of volatile-based sensors such as e-noses include operational simplicity, nondestructivity, bulk sampling (Cellini et al. 2017), and the potential to identify the original sources of VOCs and disease hotspots in the field based on volatile gradients (Oerke 2020; Oerke et al. 2014). The disadvantages are lower sensitivity and specificity compared with microbiological and molecular methods (Cellini et al. 2017), but these drawbacks might be irrelevant in comparison with optical sensors (Oerke 2020). Several commercial e-nose models have been applied for plant diagnosis purposes (Cellini et al. 2017), but challenges remain regarding sensor performance, sampling, and detection in open areas, and scaling up measurement (Cui et al. 2018). As an alternative to enoses, new small-scale portable VOC sensors have been developed that appear to be robust against environmental variation of temperature, humidity, and interference ( $\mathrm{Li}$ et al. 2019). Nevertheless, from a current perspective, VOCs may have more potential in detecting plant diseases under controlled conditions than in open fields, and VOC-based methods could possibly be suitable as secondary techniques in combination with others.

\section{Conclusions}

Interdisciplinary research involving plant science, electronics, and computer science closely tied to practical agriculture will advance plant disease detection, identification, and quantification using sensor technologies, thus improving plant health management (Mahlein 2016). An open and transparent culture of collaboration and communication among experts from different disciplines will foster and accelerate interdisciplinary research (Heim et al. 2019). Future research should include plants under different diseases and abiotic stresses to verify the uniqueness and robustness of these sensor-based signatures to detect soilborne diseases in the field. Sensor-based signatures can be potentially employed together with localized information such as

\begin{tabular}{llcl}
\multicolumn{2}{c}{ TABLE $\mathbf{2}$} \\
Comparison of the sensitivity, specificity, and potential field application (and its platform) of using spectral, thermal, and \\
volatile sensors for the detection of soilborne diseases in the field
\end{tabular}

\footnotetext{
${ }^{\mathrm{a}}$ Information partially from Oerke et al. (2014). UAV = unmanned aerial vehicle.
} 
rotation and tillage and weather conditions to provide accurate monitoring and prediction of plant disease epidemics to develop timely and efficient management practices.

\section{Acknowledgments}

We thank Drs. Maria Balota, Wade Thomason, and David McCall (members of the first author's Ph.D. committee) for their great advice on this project. We also thank Sophie LeBlanc for assistance with the VOC analysis, and Robert Wilson, Amy Taylor, and Daniel Espinosa for assisting with the greenhouse measurements.

\section{Literature Cited}

Arens, N., Backhaus, A., Döll, S., Fischer, S., Seiffert, U., and Mock, H. P. 2016. Non-invasive presymptomatic detection of Cercospora beticola infection and identification of early metabolic responses in sugar beet. Front. Plant Sci. 7:1377.

Attaran, E., Rostás, M., and Zeier, J. 2008. Pseudomonas syringae elicits emission of the terpenoid (E,E)-4,8,12-trimethyl-1,3,7,11-tridecatetraene in Arabidopsis leaves via jasmonate signaling and expression of the terpene synthase TPS4. Mol. Plant-Microbe Interact. 21:1482-1497.

Backman, P. A., and Brenneman, T. B. 1997. Stem rot. Pages 36-37 in: Compendium of Peanut Diseases, 2nd Ed. N. Kokalis-Burelle, D. M. Porter, R. Hodrfguez-Kabana, D. H. Smith, and P. Subrahmanyam, eds. American Phytopathological Society Press, St. Paul, MN.

Bock, C. H., Poole, G. H., Parker, P. E., and Gottwald, T. R. 2010. Plant disease severity estimated visually, by digital photography and image analysis, and by hyperspectral imaging. CRC Crit. Rev. Plant Sci. 29:59-107.

Boué, S. M., Shih, B. Y., Carter-Wientjes, C. H., and Cleveland, T. E. 2003. Identification of volatile compounds in soybean at various developmental stages using solid phase microextraction. J. Agric. Food Chem. 51:4873-4876.

Breiman, L. 2001. Random forests. Mach. Learn. 45:5-32.

Bruehl, G. W. 1987. Soilborne Plant Pathogens. Macmillan, New York, NY.

Cardoza, Y. J., Alborn, H. T., and Tumlinson, J. H. 2002. In vivo volatile emissions from peanut plants induced by simultaneous fungal infection and insect damage. J. Chem. Ecol. 28:161-174.

Cellini, A., Blasioli, S., Biondi, E., Bertaccini, A., Braschi, I., and Spinelli, F. 2017. Potential applications and limitations of electronic nose devices for plant disease diagnosis. Sensors 17:2596.

Chaerle, L., and Van Der Straeten, D. 2000. Imaging techniques and the early detection of plant stress. Trends Plant Sci. 5:495-501.

Cui, S., Ling, P., Zhu, H., and Keener, H. M. 2018. Plant pest detection using an artificial nose system: A review. Sensors 18:378.

Demetriades-Shah, T. H., Steven, M. D., and Clark, J. A. 1990. High resolution derivative spectra in remote sensing. Remote Sens. Environ. 33:55-64.

Divya Rani, V., and Sudini, H. 2013. Management of soilborne diseases in crop plants: An overview. Int. J. Plant, Anim. Environ. Sci. 3:156-164.

Fang, Y., and Ramasamy, R. P. 2015. Current and prospective methods for plant disease detection. Biosensors 5:537-561.

Febrero-Bande, M., and Oviedo de la Fuente, M. 2012. Statistical computing in functional data analysis: The $\mathrm{R}$ package fda.usc. J. Stat. Softw. $51: 1-28$.

Fravel, D. R., Connick, W. J., Grimm, C. C., and Lloyd, S. W. 2002. Volatile compounds emitted by sclerotia of Sclerotinia minor, Sclerotinia sclerotiorum, and Sclerotium rolfsii. J. Agric. Food Chem. 50:3761-3764.

Genuer, R., Poggi, J.-M., and Tuleau-Malot, C. 2015. VSURF: An R package for variable selection using random forests. R J. 7:19-33.

Gold, K. M., Townsend, P. A., Chlus, A., Herrmann, I., Couture, J. J., Larson, E. R., and Gevens, A. J. 2020a. Hyperspectral measurements enable pre-symptomatic detection and differentiation of contrasting physiological effects of late blight and early blight in potato. Remote Sens. 12:286.

Gold, K. M., Townsend, P. A., Larson, E. R., Herrmann, I., and Gevens, A. J. 2020b. Contact reflectance spectroscopy for rapid, accurate, and nondestructive Phytophthora infestans clonal lineage discrimination. Phytopathology 110:851-862.

Heim, R. H., Carnegie, A. J., and Zarco-Tejada, P. J. 2019. Breaking down barriers between remote sensing and plant pathology. Trop. Plant Pathol. 44:398-400.

Heim, R. H. J., Wright, I. J., Chang, H. C., Carnegie, A. J., Pegg, G. S., Lancaster, E. K., Falster, D. S., and Oldeland, J. 2018. Detecting myrtle rust (Austropuccinia psidii) on lemon myrtle trees using spectral signatures and machine learning. Plant Pathol. 67:1114-1121.

Hillnhütter, C., Mahlein, A. K., Sikora, R. A., and Oerke, E. C. 2011. Remote sensing to detect plant stress induced by Heterodera schachtii and Rhizoctonia solani in sugar beet fields. Field Crops Res. 122:70-77.

Hillnhütter, C., and Mahlein, A. K. 2008. Early detection and localization of sugar beet diseases: New approaches. Gesunde Pflanz. 60:143-149.

Katan, J. 2017. Diseases caused by soilborne pathogens: Biology, management and challenges. J. Plant Pathol. 99:305-315.

Kuhn, M., Wing, J., Weston, S., Williams, A., Keefer, C., Engelhard, A., Cooper, T., Mayer, Z., Kenkel, B., R Core Team, Benesty, M., Lescarbeau, R., Ziem, A., Scrucca, L., Tang, Y., Candan, C., and Hunt, T. 2017. CARET: Classification and regression training. $\mathrm{R}$ package version 6.0-76. Available at https://cran.r-project.org/web/packages/caret/index. html. Accessed December 14, 2019.

Li, Z., Paul, R., Tis, T. B., Saville, A. C., Hansel, J. C., Yu, T., Ristaino, J. B., and Wei, Q. 2019. Non-invasive plant disease diagnostics enabled by smartphone-based fingerprinting of leaf volatiles. Nat. Plants 5:856-866.

Liakos, K. G., Busato, P., Moshou, D., Pearson, S., and Bochtis, D. 2018. Machine learning in agriculture: A review. Sensors 18:2674.

Mahlein, A. K. 2016. Plant disease detection by imaging sensors - Parallels and specific demands for precision agriculture and plant phenotyping. Plant Dis. 100:241-251

Mahlein, A. K., Kuska, M. T., Behmann, J., Polder, G., and Walter, A. 2018. Hyperspectral sensors and imaging technologies in phytopathology: State of the art. Annu. Rev. Phytopathol. 56:535-558.

Mahlein, A. K., Oerke, E. C., Steiner, U., and Dehne, H. W. 2012. Recent advances in sensing plant diseases for precision crop protection. Eur. J. Plant Pathol. 133:197-209.

Mahlein, A. K., Rumpf, T., Welke, P., Dehne, H. W., Plümer, L., Steiner, U., and Oerke, E. C. 2013. Development of spectral indices for detecting and identifying plant diseases. Remote Sens. Environ. 128:21-30.

Moran, M. S., Inoue, Y., and Barnes, E. M. 1997. Opportunities and limitations for image-based remote sensing in precision crop management. Remote Sens. Environ. 61:319-346.

Nutter, F. W., Jr. 1990. Remote sensing and image analysis for crop loss assessment. Pages 93-105 in: Crop Loss Assessment in Rice. International Rice Research Institute, Manila, the Philippines.

Nutter, F. W., Jr., Gleason, M. L., Jenco, J. H., and Christians, N. L. 1993. Assessing the accuracy, intra-rater repeatability, and inter-rater reliability of disease assessment systems. Phytopathology 83:806-812

Nutter, F. W., Jr., Teng, P. S., and Shokes, F. M. 1991. Disease assessment terms and concepts. Plant Dis. 75:1187-1188.

Nutter, F. W., van Rij, N., Eggenberger, S. K., and Holah, N. 2010. Spatial and temporal dynamics of plant pathogens. Pages 27-50 in: Precision Crop Protection-The Challenge and Use of Heterogeneity. Springer, Dordrecht, the Netherlands.

Oerke, E. C. 2006. Crop losses to pests. J. Agric. Sci. 144:31-43.

Oerke, E. C. 2020. Remote sensing of diseases. Annu. Rev. Phytopathol. $58: 225-252$

Oerke, E. C., Mahlein, A. K., and Steiner, U. 2014. Proximal sensing of plant diseases. Pages 55-58 in: Detection and Diagnostics of Plant Pathogens. Springer, Dordrecht, the Netherlands.

Oerke, E. C., and Steiner, U. 2010. Potential of digital thermography for disease control. Pages 167-182 in: Precision Crop Protection-The Challenge and Use of Heterogeneity. Springer, Dordrecht, the Netherlands.

Pinter, P. J., Stanghellini, M. E., Reginato, R. J., Idso, S. B., Jenkins, A. D., and Jackson, R. D. 1979. Remote detection of biological stresses in plants with infrared thermometry. Science 205:585-587.

Powell, N. L., Porter, D. M., and Pettry, D. E. 1976. Use of aerial photography to detect diseases in peanut fields I. Sclerotinia blight. Peanut Sci. 3:21-24.

Punja, Z. K. 1985. The biology, ecology, and control of Sclerotium rolfsii. Annu. Rev. Phytopathol. 23:97-127.

Raza, M. M., Harding, C., Liebman, M., and Leandro, L. F. 2020. Exploring the potential of high-resolution satellite imagery for the detection of soybean sudden death syndrome. Remote Sens. 12:1213.

Samuel, A. L. 1959. Some studies in machine learning using the game of checkers. IBM J. Res. Develop. 3:210-229.

Schmitz, A., Kiewnick, S., Schlang, J., and Sikora, R. A. 2004. Use of high resolution digital thermography to detect Heterodera schachtii infestation in sugar beets. Commun. Agric. Appl. Biol. Sci. 69:359-363. 
Shokes, F. M., Róźalski, K., Gorbet, D. W., Brenneman, T. B., and Berger, D. A. 1996. Techniques for inoculation of peanut with Sclerotium rolfsii in the greenhouse and field. Peanut Sci. 23:124-128.

Singh, A., Ganapathysubramanian, B., Singh, A. K., and Sarkar, S. 2016. Machine learning for high-throughput stress phenotyping in plants. Trends Plant Sci. 21:110-124.

Tholl, D., Sohrabi, R., Huh, J.-H., and Lee, S. 2011. The biochemistry of homoterpenes - Common constituents of floral and herbivore-induced plant volatile bouquets. Phytochemistry 72:1635-1646.

Tu, J. C., and Tan, C. S. 1985. Infrared thermometry for determination of root rot severity in beans. Phytopathology 75:840-844.

Wang, T., Thomasson, J. A., Isakeit, T., Yang, C., and Nichols, R. L. 2020a. A plant-by-plant method to identify and treat cotton root rot based on UAV remote sensing. Remote Sens. 12:2453.

Wang, T., Thomasson, J. A., Yang, C., Isakeit, T., and Nichols, R. L. 2020b. Automatic classification of cotton root rot disease based on UAV remote sensing. Remote Sens. 12:1310.
Wei, X., Aguilera, M., Li, S., Langston, D. B., Jr., and Mehl, H. L. 2020. Identification of sensor-based signatures of peanut infection with Athelia rolfsii using machine learning. (Abstr.). Phytopathology 110:S2.207.

Wei, X., Langston, D. B., Jr., and Mehl, H. L. 2019. Spectral and thermal signatures of early infection of peanut with Sclerotium rolfsii. (Abstr.) Phytopathology 109:S2.1.

Wei, X., Langston, D. B., Jr., and Mehl, H. L. 2021. Spectral and thermal responses of peanut to the infection and colonization with Athelia rolfsii. PhytoFrontiers 1:173-181.

Zarco-Tejada, P. J., Camino, C., Beck, P. S. A., Calderon, R., Hornero, A., Hernández-Clemente, R., Kattenborn, T., Montes-Borrego, M., Susca, L., Morelli, M., Gonzalez-Dugo, V., North, P. R. J., Landa, B. B., Boscia, D., Saponari, M., and Navas-Cortes, J. A. 2018. Previsual symptoms of Xylella fastidiosa infection revealed in spectral plant-trait alterations. Nat. Plants 4:432-439.

Zhang, Z. M., Wu, W. W., and Li, G. K. 2008. A GC-MS study of the volatile organic composition of straw and oyster mushrooms during maturity and its relation to antioxidant activity. J. Chromatogr. Sci. 46:690-696. 